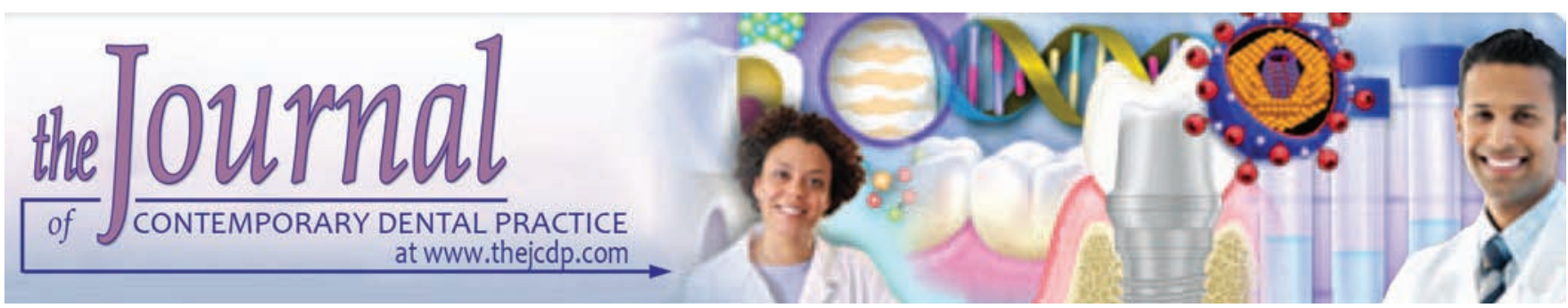

\title{
Current prescribing Practices for Antibiotic Prophylaxis: A Survey of Dental Practitioners
}

\author{
${ }^{1}$ Leah S Spittle, ${ }^{2}$ Kathleen B Muzzin, ${ }^{3}$ Patricia R Campbell, ${ }^{4}$ Janice P DeWald, ${ }^{5}$ Francisco Rivera-Hidalgo
}

\begin{abstract}
Aim: Antibiotic prophylaxis guidelines have been developed for health care and dentistry. The authors examined whether dentists were following the 2007 American Heart Association (AHA) and the 2012 American Association of Orthopaedic Surgeons (AAOS) recommendations for antibiotic prophylaxis.
\end{abstract}

Materials and methods: A survey was sent to 600 dentists in the south-central region of the United States. The survey examined dentists' prescribing practices for patients with cardiac and prosthetic joint replacements, antibiotic regimen prescribed, confidence in their prescription decisions, and dentistry's contribution to antibiotic resistance.

Results: The response rate was $28.7 \%(n=172)$. Dentists followed the 2007 AHA guidelines for patients with a history of infective endocarditis (95\%), and cardiac valves repaired with prosthetic materials (76\%) or animal/donor tissue (61\%). For prosthetic joint replacement, $72 \%$ prescribed antibiotic prophylaxis within the first 2 years after placement and $58 \%$ continued to prescribe after 2 years. Amoxicillin was the most frequently prescribed antibiotic for cardiac conditions (92\%) and prosthetic joint replacements (77\%). No significant differences were found in relation to dentists' gender $(p=0.75)$, year of graduation $(p=0.71)$, scope of practice $(p=0.30)$, and their confidence in their prescription decisions; $73 \%$ believed taking multiple doses of antibiotics leads to antibiotic resistance.

Conclusion: The majority of dentists were following the 2007 AHA guidelines. Confusion regarding the 2012 AAOS guidelines for prosthetic joint replacement was evident.

Clinical significance: Keeping current with changing antibiotic prophylaxis guidelines ensures dentists are providing the best evidence-based dentistry for their patients.

\footnotetext{
${ }^{1-4}$ Caruth School of Dental Hygiene, Texas A\&M University College of Dentistry, Dallas, Texas, USA

${ }^{5}$ Department of Periodontics, College of Dentistry, Texas A\&M University, Dallas, Texas, USA

Corresponding Author: Kathleen B Muzzin, Caruth School of Dental Hygiene, Texas A\&M University, College of Dentistry Dallas, Texas, USA, e-mail: kmuzzin@tamhsc.edu
}

Keywords: Antibiotic prophylaxis, Bacterial endocarditis, Dentists, Practice guidelines.

How to cite this article: Spittle LS, Muzzin KB, Campbell PR, DeWald JP, Rivera-Hidalgo F. Current prescribing Practices for Antibiotic Prophylaxis: A Survey of Dental Practitioners. J Contemp Dent Pract 2017;18(7):559-566.

Source of support: This study was funded by an Intramural Grant from Texas A\&M Health Science Center. Texas A\&M Health Science Center had no role in the study design; collection management, analysis, and interpretation of data; or preparation, review, or approval of the manuscript.

Conflict of interest: None

\section{INTRODUCTION}

Over the years, investigators have focused on invasive dental procedures as a potential point of entry for bacteria to enter the bloodstream. ${ }^{1-7}$ Transient bacteremias may occur that could potentially colonize on vulnerable areas within the heart and joints repaired with prosthetic devices. As a result, premedication with antibiotics before dental treatment has been recommended by the American Heart Association (AHA) and the American Academy of Orthopaedic Surgeons (AAOS) ${ }^{8-10}$ However, controversy concerning the efficacy and safety of antibiotic prophylaxis has been under debate for many decades. ${ }^{11-13}$ Current evidence suggests that dental procedures play a minor role in the development of infective endocarditis and periprosthetic joint infection. ${ }^{14-19}$ Increased awareness of antibiotic resistance has also questioned the appropriateness and scope of antibiotic prophylaxis use in dentistry.

Evolving scientific evidence has led the AHA and AAOS to make numerous changes in their recommendations for antibiotic prophylaxis. ${ }^{8-10,20,21}$ The American Dental Association (ADA) has worked closely with both organizations and has approved their recommendations for antibiotic prophylaxis as they relate to dentistry. ${ }^{21,22}$ 
Changes in the 2007 AHA and 2012 AAOS antibiotic prophylaxis guidelines have received mixed reactions from health care providers, and studies have reported wide variations in how practitioners are prescribing antibiotic prophylaxis. ${ }^{23-29}$

Lauber et $\mathrm{al}^{23}$ surveyed Canadian dentists and family physicians and found that dentists were more familiar with the 2007 AHA antibiotic prophylaxis protocols than physicians in selecting the correct "first-line" antibiotic for prophylaxis. Jain et $\mathrm{al}^{24}$ found that the majority of Canadian dentists (89.2\%) and dental hygienists (89.9\%) deferred antibiotic prophylaxis prescription decisions to the patient's cardiologist. Another Canadian study found that $77 \%$ of orthopedic surgeons and $71 \%$ of dental surgeons routinely prescribe antibiotic prophylaxis for patients with total joint replacement, even though the 2012 AAOS guidelines no longer recommend routine prophylactic coverage. $^{25}$

Tong et $\mathrm{al}^{26}$ surveyed Singapore dentists and found that $39.8 \%$ followed the 2007 AHA guidelines and could accurately identify which dental procedures required antibiotic prophylaxis. However, $30.2 \%$ of the dentists reported their prescribing practices were based solely on the

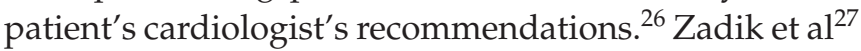
surveyed Israeli dentists and found that the majority of dentists $(81.3 \%)$ identified the cardiac conditions that require antibiotic prophylaxis. However, $42 \%$ of the dentists stated they would prescribe antibiotic prophylaxis for patients with mitral valve prolapse (MVP) with regurgitation, which implied that some Israeli dentists were still following the 1997 AHA guidelines. ${ }^{27}$

Coutinho et $\mathrm{al}^{28}$ examined the knowledge and practices of dentists in preventing infective endocarditis in children. Of the 21 dentists, 33\% stated they followed the 2007 AHA antibiotic prophylaxis guidelines. ${ }^{28}$ A total of $33 \%$ of dentists stated they had offered an explanation about the risk associated with bacteremia and the patient's inadequate oral health. ${ }^{28}$ In addition, $14 \%$ of dentists felt there was not enough time to discuss with the patient risks $v s$ benefits of antibiotic prophylaxis. ${ }^{28}$ Finally, to avoid the overuse of antibiotics, 33\% stated they performed multiple dental procedures during one appointment. ${ }^{28}$

Lockhart et $\mathrm{al}^{29}$ surveyed US dentists regarding their compliance with the 2007 AHA recommendations for antibiotic prophylaxis. The majority of the dentists (70\%) stated they had patients in their practice who took antibiotic prophylaxis before dental treatment even though the 2007 AHA guidelines no longer recommended it; these included ventricular septal defect, atrial septal defect, hypertrophic cardiomyopathy, rheumatic heart disease, aortic or mitral stenosis, and bicuspid valve disease. ${ }^{29}$ They also found that $57 \%$ of the patients' prescriptions for antibiotic prophylaxis came from the patient's physician and $33 \%$ were due to patient preference. ${ }^{29}$

At present, there is limited research on US dental practitioners' antibiotic prophylaxis prescribing practices. Therefore, we chose to examine whether dentists in the south-central region of the United States were (1) following the 2007 AHA, as well as the 2012 AAOS antibiotic prophylaxis guidelines, (2) involving the patient in the decision to take antibiotic prophylaxis, (3) confident in their prescription decisions for antibiotic prophylaxis, (4) concerned that dentistry is indirectly contributing to antibiotic resistance, and (5) confused about the appropriate use of antibiotic prophylaxis.

\section{MATERIALS AND METHODS}

The questionnaire for our investigation was a modified version of a survey developed by Tong et al. ${ }^{26}$ Our survey consisted of 25 questions containing multiple choice, yes/no response, and space for additional comments. Questions included demographics, current antibiotic prophylaxis prescribing practices, and tables in which the respondents identified various health conditions and medical and dental treatments for which they would prescribe antibiotic prophylaxis. We asked which antibiotic prophylaxis regimen they followed, as well as questions regarding confidence in their prescribing decisions and opinions regarding antibiotic resistance. We pilot tested the survey among three dental specialists and used their responses to modify the survey as needed. We submitted the research proposal to the Institutional Review Board at Texas A\&M Health Science Center, Baylor College of Dentistry, which granted an expedited status (2015-0406BCD-EXP) on July 8, 2015.

Our sample population consisted of a randomized sample of dentists from the south-central states of Texas, Oklahoma, and Kansas. We contacted each state dental board and requested a current list of licensed dentist's addresses. From each state 200 dental practitioners were randomly selected. Our sample population was based on a 50\% response rate, and to reduce error a $95 \%$ confidence interval was used. Each selected dentist received a cover letter that explained the study, an invitation to participate, a survey with a tracking number, and a stamped return envelope. After 4 weeks, we sent all nonresponders a second cover letter, an additional survey, and another stamped return envelope. Completion of the survey served as the dentists' consent to participate in our study.

Some knowledge and practice-based questions contained "right or wrong" answers, and for those questions, we created a scoring system to analyze the data. If the question was regarding a heart condition, the 2007 AHA guidelines were used. If the question was in regard to 
prosthetic joint replacement, the 2012 AAOS guidelines were used. Correct answers were awarded one point. If the respondent did not follow the guidelines, a point was taken away. If the respondent selected "per physician request" (which we considered neither right nor wrong), a score of zero was given. Any survey that had four or more responses left blank was considered "nonresponsive" and was excluded from the data analysis. Only the most prominent cardiac and orthopedic findings noted in the survey were analyzed.

Statistical analysis included descriptive statistics, t-tests, and weighted logistic regression. A p-value $\leq 0.05$ was considered statistically significant. Excel data analysis tool Pak was used to conduct t-tests to determine whether the year the dentist graduated from dental school affected their knowledge of the 2007 AHA antibiotic guidelines. Statistical software SAS 9.4, copyright 2012, was used for weighted logistic regression analysis and was used to determine whether demographic variables affected the dentist's confidence in their prescription decisions.

\section{RESULTS}

Of the 600 surveys mailed, 29 were undeliverable. From the total sample population of $n=600$, the response rate was $28.7 \%(n=172)$. The number of responses for each question may vary since not all respondents answered every question. Table 1 depicts the dentist's years in practice, scope of practice, gender, and year of graduation.

The dentists were asked which guidelines they routinely followed to determine the need for antibiotic prophylaxis. The majority of dentists $(53.0 \%, \mathrm{n}=97)$ stated they followed the 2007 ADA guidelines (which align with the AHA). Approximately $36.0 \%(n=65)$ of the dentists reported that they followed the patient's physician/
Table 1: Demographics of dentists

\begin{tabular}{ll}
\hline Parameter & $n(\%)$ \\
\hline Years in practice & \\
$0-3$ & $14(8.1)$ \\
$4-7$ & $16(9.3)$ \\
$8-11$ & $22(12.8)$ \\
$12-15$ & $10(5.8)$ \\
$16-20$ & $108(62.8)$ \\
Missing & $2(1.2)$ \\
Primary area of practice & \\
General dentistry & $127(73.8)$ \\
Oral surgery & $9(5.2)$ \\
Periodontics & $8(4.7)$ \\
Other & $27(15.7)$ \\
Missing & $1(0.6)$ \\
Gender & \\
Male & $132(76.7)$ \\
Female & $37(21.5)$ \\
No answer & $3(1.7)$ \\
Year of graduation* & \\
Before 1980 & $45(27.4)$ \\
1980-1989 & $38(23.2)$ \\
1990-1999 & $24(14.6)$ \\
2000-2009 & $36(22.0)$ \\
2010 + & $21(12.8)$ \\
\hline
\end{tabular}

*Sum of responses may be $<172$ since "nonresponsive" dentists were excluded

specialist order or recommendation as their guideline. Of those dentists who reported "other," $11.0 \%(n=21)$ responded that they followed the 2007 AHA guidelines.

Graph 1 displays the cardiac conditions and treatments recommended by the 2007 AHA guidelines for antibiotic prophylaxis. Patients with a previous history of infective endocarditis and prosthetic heart valves were correctly identified by the majority of dentists as needing antibiotic prophylaxis. However, only one-third of the dentists were prescribing antibiotic prophylaxis

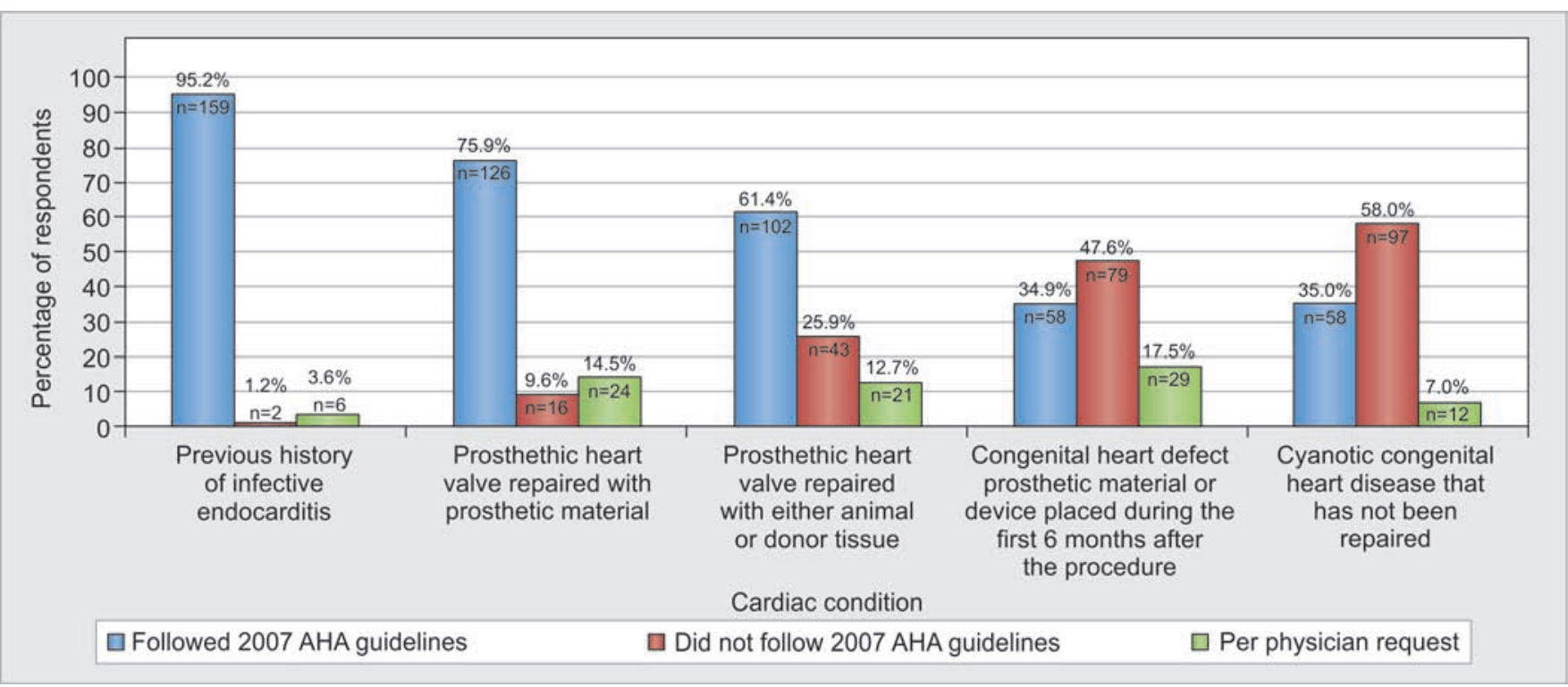

Graph 1: Prescribing practices for cardiac conditions that require antibiotic prophylaxis 


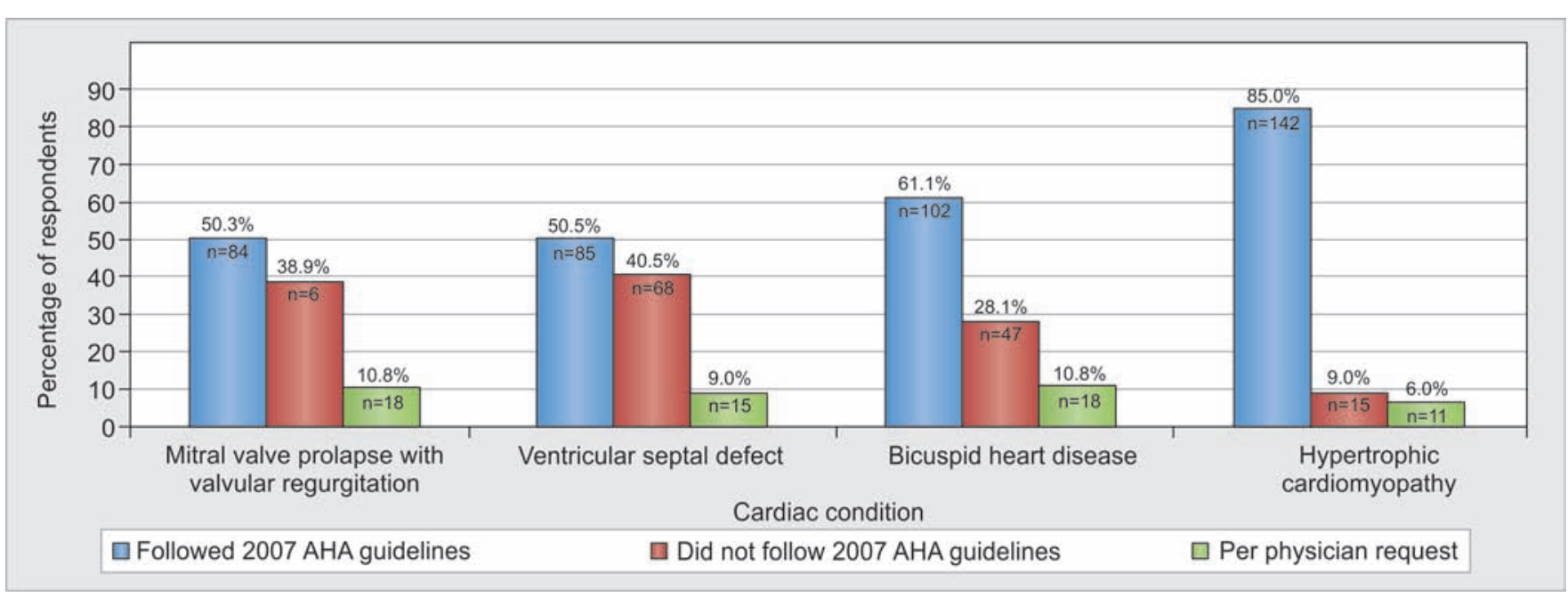

Graph 2: Prescribing practices for cardiac conditions that no longer require antibiotic prophylaxis

appropriately for patients with a congenital heart defect prosthetic material or device placed during the first 6 months after the procedure and cyanotic congenital heart disease that had not been repaired. Graph 2 displays the cardiac conditions that do not require antibiotic prophylaxis. Approximately $50 \%$ of the dentists were no longer prescribing antibiotics for patients with MVP with valvular regurgitation or for ventricular septal defect, while a higher percentage of dentists were no longer prescribing antibiotic prophylaxis for bicuspid heart disease (61.1\%) and hypertrophic cardiomyopathy (85\%). In regard to prosthetic joint replacement, we found that $71.7 \%(n=119)$ of dentists chose to prescribe antibiotic prophylaxis for patients with a total prosthetic joint replacement within the past 2 years and $57.8 \%(n=96)$ chose to prescribe antibiotic prophylaxis for patients who had a total prosthetic joint replacement for 2 years or longer.

Graph 3 displays the dental procedures that may involve bleeding, and we found that the majority of dentists chose to prescribe antibiotic coverage for these procedures. However, approximately $51.0 \%$ of the dentists would not prescribe antibiotic prophylaxis before laser periodontal therapy. Table 2 lists the antibiotic prophylaxis regimen that was prescribed by the dentists. The most frequently prescribed antibiotic for cardiac patients not allergic to penicillin was amoxicillin $2 \mathrm{gm}$ orally 1 hour before procedure (91.2\%). To prevent transient bacteremia for patients with total joint replacement, $77.2 \%$ of the dentists prescribed amoxicillin 2 gm orally 1 hour before dental treatment, $10.8 \%$ prescribed amoxicillin 2 gm orally 1 hour before procedure, then 1 gm 6 hours after the initial dose, and $4.4 \%$ chose to prescribe $2 \mathrm{gm}$ of oral cephalexin 1 hour before dental treatment.

A t-test was performed to determine whether the year the dentist graduated from dental school affected their knowledge of the 2007 AHA antibiotic guidelines. An F-test was performed and determined that unequal variances were present between the respondents who

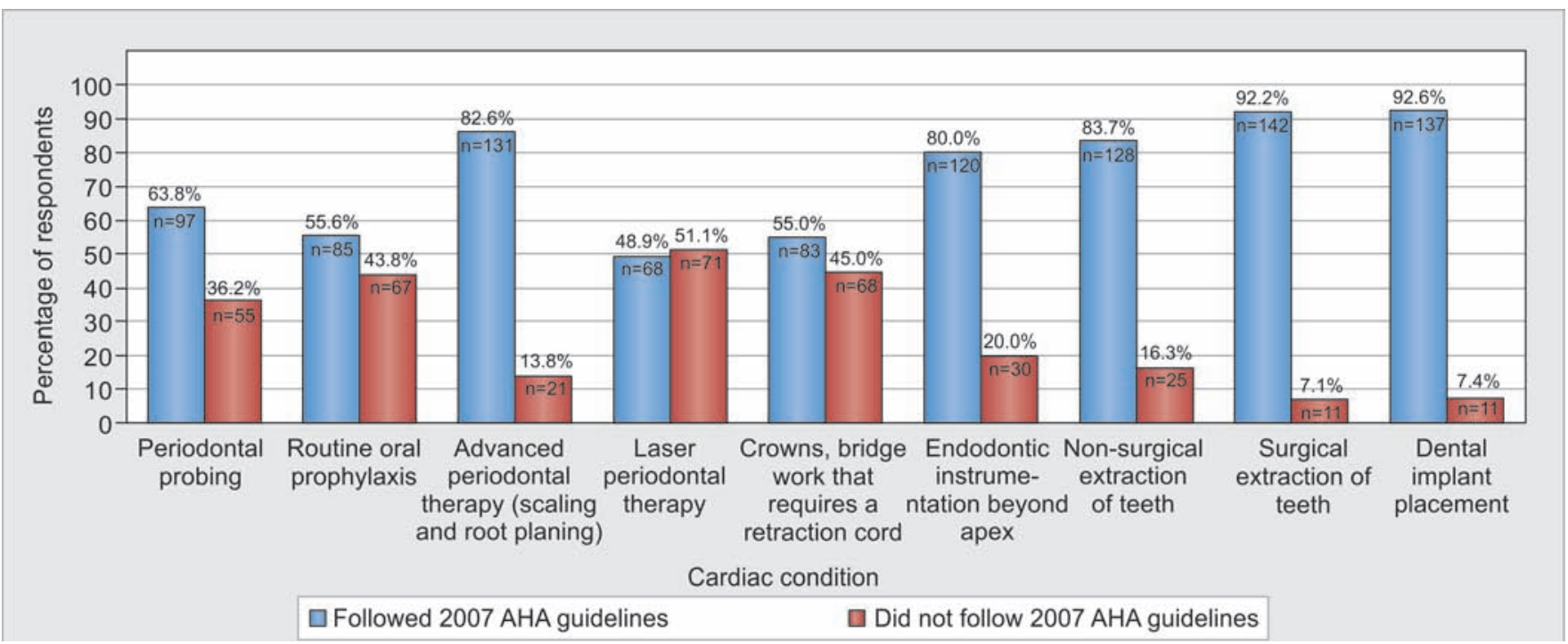

Graph 3: Prescribing practices for dental procedures that may involve bleeding and require antibiotic prophylaxis 
Current prescribing Practices for Antibiotic Prophylaxis: A Survey of Dental Practitioners

Table 2: Antibiotic prophylaxis regimen dentists prescribed for patients who had a cardiac condition or prosthetic joint replacement

\begin{tabular}{|c|c|}
\hline Parameter & $n(\%)$ \\
\hline \multicolumn{2}{|l|}{ Cardiac conditions - not allergic to amoxicillin* } \\
\hline Amoxicillin 2 gm orally 1 hour before procedure & $156(91.2)$ \\
\hline Amoxicillin 2 gm orally 1 hour before procedure, then $1 \mathrm{gm} 6$ hours after initial dose & $9(5.2)$ \\
\hline Penicillin VK 2 gm orally 1 hour before procedure, then $1 \mathrm{gm} 6$ hours after initial dose & $1(0.6)$ \\
\hline Other & $5(3.0)$ \\
\hline \multicolumn{2}{|l|}{ Cardiac conditions - allergic to amoxicillin* } \\
\hline Clindamycin $600 \mathrm{mg}$ orally 1 hour before procedure & $133(78.2)$ \\
\hline Cephalexin 2 gm orally 1 hour before procedure & $27(15.9)$ \\
\hline Azithromycin $500 \mathrm{mg}$ orally 1 hour before procedure & $7(4.1)$ \\
\hline Other & $3(1.8)$ \\
\hline \multicolumn{2}{|l|}{ Prosthetic joint replacement* } \\
\hline Amoxicillin 2 gm orally 1 hour before procedure & $122(77.2)$ \\
\hline Amoxicillin 2 gm orally 1 hour before procedure, then $1 \mathrm{gm} 6$ hours after initial dose & $17(10.8)$ \\
\hline Cephalexin 2 gm orally 1 hour before procedure & $7(4.4)$ \\
\hline Penicillin VK gm orally 1 hour before procedure; then $1 \mathrm{gm} 6$ hours after initial dose & $1(0.6)$ \\
\hline Other & $11(7.0)$ \\
\hline
\end{tabular}

*Sum of responses may be $<172$ since "nonresponsive" dentists were excluded

Table 3: t-test analyzing how well dentists followed 2007 AHA guidelines based on year of graduation from dental school

\begin{tabular}{llll}
\hline $\begin{array}{l}\text { Graduation } \\
\text { year }\end{array}$ & Mean \pm SD & $\begin{array}{l}\text { 95\% confidence } \\
\text { interval }\end{array}$ & $p$-value \\
\hline Pre-1990 & $31.03 \pm 10.89$ & $29.70-32.36$ & $<0.001^{\dagger}$ \\
Post-1990 & $36.57 \pm 8.05$ & $35.62-37.54$ & \\
\hline $\begin{array}{l}\text { SD: Standard deviation; }{ }^{\dagger} \mathrm{p}(\mathrm{T} \leq \mathrm{t}) \\
\text { variances }\end{array}$ & two-tailed $t$-test for unequal
\end{tabular}

graduated before 1990 and after 1990. Therefore, a t-test for unequal variances was conducted and found that the dentists who graduated after 1990 did significantly better in following the 2007 AHA guidelines than earlier graduates $(\mathrm{p}=0.001)$ (Table 3$)$.

When dentists were asked if the patient was an integral part of the decision-making process when discussing an antibiotic prophylaxis regimen, the majority of dentists $(72.4 \%, n=123)$ said "yes." However, $79.5 \%(n=136)$ of the dentists did not provide educational literature on antibiotic prophylaxis. If a patient refused to take the recommended antibiotic prophylaxis, $53.2(\mathrm{n}=82)$ of the dentists stated they would not treat the patient. The majority of the dentists $(89.0 \%, \mathrm{n}=151)$ also stated that they do not make their patients sign an informed consent that discusses the risk $v s$ benefit of antibiotic prophylaxis.

Approximately $80.0 \%(n=129)$ of the dentists stated they felt confident in their personal prescription decisions in regard to antibiotic prophylaxis and preventing infective endocarditis. Further analysis was conducted to determine if demographics were related to their confidence in prescribing antibiotic prophylaxis. Weighted logistic regression showed no significant differences in relation to gender $(p=0.75)$, year graduating from dental school ( $\mathrm{p}=0.71)$, and primary area of practice $(\mathrm{p}=0.30)$ and the dentist's confidence level when prescribing antibiotic prophylaxis.
When asked if they believed there is a risk for antibiotic resistance if a patient is taking a required antibiotic prophylaxis regimen for each dental visit, the majority of dentists $(73.2 \%, \mathrm{n}=120)$ stated "yes." The majority of dentists $(72.0 \%, \mathrm{n}=120)$ also believed that antibiotic prophylaxis promotes the emergence of antibiotic-resistant microorganisms.

When asked if they believed there was confusion regarding antibiotic prophylaxis protocols used in dentistry, 92.0\% ( $n=155)$ of the dentists answered "yes". Anecdotal written comments included several dentists stating they provided antibiotic prophylaxis to avoid a lawsuit regardless of what the guidelines recommended, as well as dentists and physicians not agreeing what conditions should be covered with antibiotic prophylaxis.

\section{DISCUSSION}

This study examined the antibiotic prophylaxis prescribing practices of dentists in the south-central region of the United States and found that $53 \%$ of the respondents followed the 2007 AHA recommendations as they pertain to dentistry. These results are encouraging as they are higher than the percentage of dentists determined to be following the 2007 AHA guidelines by researchers Tong et $\mathrm{al}^{26}(40 \%)$ and Coutinho et $\mathrm{al}^{28}(33 \%)$. However, our study found that $36 \%$ of dentists preferred to follow the patient's physician/specialist orders. The previous research has found that dentists often consult with physicians for advice on the need for antibiotic prophylaxis for their patients. ${ }^{24,26,28,29}$ Studies have also found some patients are reluctant to stop taking antibiotic prophylaxis even when it is no longer needed and insist that their dentist consult with their physician before receiving care. ${ }^{24,26,29}$ Unfortunately, some dentists may have received conflicting antibiotic prophylaxis 
recommendations by the patient's physician. ${ }^{30}$ According to Tong and Rothwell, ${ }^{30}$ this becomes an ethical dilemma for dental practitioners because they may feel obligated to prescribe an antibiotic regimen that does not align with current antibiotic guidelines. The dentist is ultimately responsible for his/her patient's welfare during dental treatment, and in these instances, the dentist should personally contact the physician to discuss the best treatment for the patient.

Dentists in our study demonstrated they understood most of the cardiac risk factors and principles underlying the 2007 AHA guidelines. However, 48\% of dentists in our study would not prescribe antibiotic prophylaxis for patients with a congenital heart defect repaired with prosthetic material or device placed during the first 6 months after the procedure. This is considered a highrisk cardiac condition, is uncommon, and, therefore, may explain why the dentists in our study chose not to provide antibiotic prophylactic coverage.

Unlike previous research, the dentists in our study were not prescribing antibiotic prophylaxis for patients with valvular disease independent of regurgitation, hypertrophic cardiomyopathy, ventricular septal defect, or bicuspid valve disease. ${ }^{27,29}$ According to the 2007 AHA guidelines, these cardiac conditions no longer need to be covered prophylactically with antibiotics before dental treatment. However, some dentists in our study continue to prescribe antibiotic prophylaxis for these low- to moderate-risk cardiac conditions. Tong et $\mathrm{al}^{26}$ stated that the reason many dentists continue to follow outdated guidelines is their reliance on old prescribing habits or their reliance on their memory when making prescription decisions. Uncertainty by dentists may also lead them to prescribe prophylactic antibiotics as a "safety net."19,30 To avoid uncertainty, Jain et $\mathrm{al}^{24}$ suggest that dental practitioners should keep the 2007 AHA guidelines somewhere readily accessible or incorporate them into the electronic health record.

In 2012, the AAOS in conjunction with the ADA recommended that "practitioners might consider discontinuing the practice of routinely prescribing prophylactic antibiotics for patients with hip and knee prosthetic joint implants undergoing dental procedures. ${ }^{21}$ Dental practitioners voiced concerns regarding the guidelines and requested further clarification from the ADA. In 2014, the ADA formed a panel and found that the current evidence "failed to demonstrate an association between dental procedures and prosthetic joint infection. ${ }^{31 " ~ H o w e v e r, ~ i t ~}$ appears that the dentists in our study were still following either the 2003 AAOS / ADA (72\%) or the 2009 AAOS (58\%) antibiotic prophylaxis guidelines. This could be due to dentists receiving conflicting recommendations from the patient's orthopedic surgeon, or they were uncomfortable providing dental treatment for patients with total joint replacements without antibiotic coverage. ${ }^{19,25}$ According to the ADA, antibiotic prophylaxis should be based on the patient's medical history and the evaluation of specific comorbidities. ${ }^{31}$

The AHA has outlined the appropriate antibiotic and dosage for patients with high-risk cardiac conditions. This includes amoxicillin $2 \mathrm{gm}$ or clindamycin $600 \mathrm{gm}$ for patients allergic to amoxicillin. The results of our study found that the dentists were following the 2007 AHA antibiotic prophylaxis dosing regimens, which support the findings by Lauber et al. ${ }^{23}$ It also appears that dentists in our study were referring to the 2007 AHA antibiotic regimen recommendations for total joint replacements. Only $4.4 \%$ chose cephalexin, 2 gm 1 hour before dental treatment, which is the first-line antibiotic recommended for patients with a total joint replacement. ${ }^{21,32,33}$

Overall, dentists in our study appear to be knowledgeable in regard to when it is appropriate to prescribe antibiotic prophylaxis for high-risk patients undergoing invasive dental procedures, such as extractions, periodontal probing, scaling and root planing, and dental prophylaxis. Tong et $\mathrm{al}^{26}$ and Zadik et $\mathrm{al}^{27}$ found similar findings stating that these procedures were clearly invasive and the dentists in their studies agreed that the patients should be covered by antibiotic prophylaxis if they were at risk for infective endocarditis. However, more than half of the dentists in this study were not prescribing antibiotic prophylaxis for laser periodontal therapy that could be considered invasive. ${ }^{34}$ Some dentists may not provide this form of periodontal therapy; therefore, they may be unaware of the possible bacteremia that can occur during treatment.

Our study found that the dentists who graduated from approximately 1990 to 2014 were more likely to follow the 2007 AHA antibiotic prophylaxis protocols (Table 3). Epstein et $\mathrm{al}^{35}$ found that recent dental graduates prescribed antibiotic prophylaxis at a lower rate than earlier graduates, suggesting they may have more knowledge on evidence-based approaches to dental care and current antibiotic prophylaxis protocols. Zadik et $\mathrm{al}^{27}$ suggest that dentists' knowledge of the 2007 AHA guidelines improved after the 1997 version because they were simplified and provided a clearer delineation for those at highest risk for developing infective endocarditis.

The dental practitioner's clinical and professional judgment, as well as the patient's preferences, should be considered together before providing antibiotic prophylaxis. ${ }^{21}$ While $72 \%$ of our respondents stated that the patient's input was an integral part of the decisionmaking process, more than half of the dentists stated that they would refuse to treat a patient if the patient did not follow the dentist's orders for antibiotic prophylaxis. These findings and attitudes could be related to lack of educational material given to patients as well as the lack 
of time to discuss the risks $v$ s benefits and the role that poor oral hygiene plays in developing a transient bacteremia. ${ }^{28}$ Depending on which health care provider they see, patients may also receive conflicting recommendations regarding antibiotic prophylaxis. The patients should be informed of the current antibiotic prophylaxis guidelines and should be advised to make their decision based on the most up-to-date information.

Approximately $80 \%$ of the dentists in our study stated they felt confident in their prescription decisions in regard to antibiotic prophylaxis and preventing infective endocarditis, while Tong et $\mathrm{al}^{26}$ found that $67 \%$ of dentists in their study felt confident. Tong et $\mathrm{al}^{26}$ also reported that there was no statistical difference between general dentists and specialists in their confidence level, which was also a finding in our study.

The broad use of antibiotic prophylaxis is no longer recommended by AHA and AAOS. Prescribing antibiotics in a judicious and prudent manner is an essential strategy to reduce the spread of antibiotic resistance. However, previous studies have found that dental practitioners have been overprescribing antibiotic prophylaxis, which suggests that there is a poor translation of the guidelines into clinical practice. ${ }^{28,36,37}$ The dentists in our study believed that there is a risk for antibiotic resistance if a patient is taking a required antibiotic prophylaxis regimen for each dental visit. Although this increased awareness of antibiotic resistance has reached the attention of health care professionals and the public, there is limited research on dentistry's contribution.

In our study, $92 \%$ of dentists stated they believed there was confusion regarding antibiotic prophylaxis protocols used in dentistry. Some dental practitioners noted a lack of agreement between dentists and physicians on which cardiac conditions should be covered as well as joint replacement. Jain et $\mathrm{al}^{24}$ reported that dentists felt the 2007 AHA guidelines regarding cardiac conditions were too vague, difficult to remember, and/or disagreed with the current recommendations. Therefore, it has been suggested that more continuing education courses on antibiotic prophylaxis should be available to dental practitioners.

Our study was limited to sampling three states which may not represent dentists nationwide. The topic of antibiotic prophylaxis could be considered controversial; therefore, some of the dentists may have chosen not to answer all the survey questions, or chose to forgo participation altogether for fear of being "inaccurate" in their responses. Future studies should include a larger sample size of dentists from varying regions in the United States. Furthermore, future research should include surveying US physicians/specialists to determine their current knowledge, practices, and attitudes regarding the 2007 AHA antibiotic prophylaxis guidelines and the 2012 AAOS recommendations in relation to dentistry.

\section{CONCLUSION}

Although the majority of the dentists in our study followed the 2007 AHA guidelines for patients with highrisk cardiac conditions, there is still confusion regarding the antibiotic protocols for total joint replacement. Our study found that the dentist's graduation year had some influence over the respondent's knowledge and practice patterns in regard to antibiotic prophylaxis. Knowledge of current antibiotic prophylaxis guidelines and education on the research related to the guidelines is imperative. Keeping up with the latest antibiotic prophylaxis protocols ensures that dentists are providing the best evidence-based dentistry to their patients.

\section{ACKNOWLEDGMENT}

Authors would like to thanks to Mr Richard Payne for his statistical assistance with this study.

\section{REFERENCES}

1. Lucas V, Roberts GJ. Odontogenic bacteremia following tooth cleaning procedures in children. Pediatr Dent 2000 Mar-Apr;22(2):96-100.

2. Lockhart PB, Brennan MT, Sasser HC, Fox PC, Paster BJ, Bahrani-Mougeot FK. Bacteremia associated with toothbrushing and dental extraction. Circulation 2008 Jun;117(24): 3118-3125.

3. Bahrani-Mougeot FK, Paster BJ, Coleman S, Ashar J, Barbuto S, Lockhart PB. Diverse and novel oral bacterial species in blood following dental procedures. J Clin Microbiol 2008 Jun;46(6):2129-2132.

4. Kinane DF, Riggio MP, Walker KF, MacKenzie D, Shearer B. Bacteraemia following periodontal procedures. J Clin Periodontol 2005 Jul;32(7):708-713.

5. Roberts GJ, Holzel HS, Sury MR, Simmons NA, Gardner P, Longhurst P. Dental bacteremia in children. Pediatr Cardiol 1997 Jan-Feb;18(1):24-27.

6. Daly C, Mitchell D, Grossberg D, Highfield J, Stewart D. Bacteraemia caused by periodontal probing. Aust Dent J 1997 Apr;42(2):77-80.

7. Zhang W, Daly CG, Mitchell D, Curtis B. Incidence and magnitude of bacteraemia caused by flossing and by scaling and root planing. J Clin Periodontol 2013 Jan;40(1):41-52.

8. Wilson W, Taubert KA, Gewitz M, Lockhart PB, Baddour LM, Levison M, Bolger A, Cabell CH, Takahashi M, Baltimore RS, et al. Prevention of infective endocarditis: guidelines from the American Heart Association: a guideline from the American Heart Association Rheumatic Fever, Endocarditis, and Kawasaki Disease Committee, Council on Cardiovascular Disease in the Young, and the Council on Clinical Cardiology, Council on Cardiovascular Surgery and Anesthesia, and the Quality of Care and Outcomes Research Interdisciplinary Working Group. Circulation 2007 Oct;116(15):1736-1754.

9. American Academy of Orthopaedic Surgeons. Antibiotic prophylaxis for bacteremia in patients with joint replacements. Rosemont (IL): AAOS Information Statement; 2009 [cited 2016 Dec 21]. Available from: http:/ / www.pacosm.com/wp/ wp-content/uploads/2015/08/Antibiotic-Prophylaxis-forTJA-pts.-AAOS-March-2009.pdf. 
10. American Dental Association, American Academy of Orthopedic Surgeons. Antibiotic prophylaxis for dental patients with total joint replacements. J Am Dent Assoc 2003 Jul;134(7):895-899.

11. Lockhart PB, Loven B, Brennan MT, Fox PC. The evidence base for the efficacy of antibiotic prophylaxis in dental practice. J Am Dent Assoc 2007 Apr;138(4):458-474.

12. Little JW, Jacobson JJ, Lockhart PB, American Academy of Oral Medicine. The dental treatment of patients with joint replacements: a position paper from the American Academy of Oral Medicine. J Am Dent Assoc 2010 Jun;141(6):667-671.

13. DeSimone DC, Tleyjeh IM, Correa de Sa DD, Anavekar NS, Lahr BD, Sohail MR, Steckelberg JM, Wilson WR, Baddour LM, Mayo Cardiovascular Infections Study Group. Incidence of infective endocarditis due to viridans group Streptococci before and after the 2007 American Heart Association's prevention guideline: an extended evaluation of the Olmstead county, Minnesota, population and nationwide inpatient sample. Mayo Clin Proc 2015 Jul;90(7):874-881.

14. Imperiale TF, Horwitz RI. Does prophylaxis prevent postdental infective endocarditis? A controlled evaluation of protective efficacy. Am J Med 1990 Feb;88(2):131-136.

15. Duval X, Alla F, Hoen B, Danielou F, Larrieu S, Delahaye F, Leport C, Briançon S. Estimated risk of endocarditis in adults with predisposing cardiac conditions undergoing dental procedures with or without antibiotic prophylaxis. Clin Infect Dis 2006 Jun;42(12):e102-e107.

16. Skaar DD, O'Connor H, Hodges JS, Michalowicz BS. Dental procedures and subsequent prosthetic joint infections: findings from the Medicare current beneficiary survey. J Am Dent Assoc 2011 Dec;142(12):1343-1351.

17. Berbari EF, Osmon DR, Carr A, Hanssen AD, Baddour LM, Greene D, Kupp LI, Baughan LW, Harmsen WS, Mandrekar JN, et al. Dental procedures as risk factors for prosthetic hip or knee infection: a hospital-based prospective case-control study. Clin Infect Dis 2010 Jan;50(1):8-16.

18. Chen PC, Tung YC, Wu PW, Wu LS, Lin YS, Chang CJ, Kung S, $\mathrm{Chu} \mathrm{PH}$. Dental procedures and the risk of infective endocarditis. Medicine (Baltimore) 2015 Oct;94(43):e1826.

19. Kao FC, Hsu YC, Chen WH, Lin JN, Lo YY, Tu YK. Prosthetic joint infection following invasive dental procedures and antibiotic prophylaxis in patients with hip or knee arthroplasty. Infect Control Hosp Epidemiol 2017 Feb;38(2):1-8.

20. Advisory statement. Antibiotic prophylaxis for dental patients with total joint replacements. American Dental Association; American Academy of Orthopaedic Surgeons. J Am Dent Assoc 1997 Jul;128(7):1004-1008.

21. American Academy of Orthopaedic Surgeons; American Dental Association. Prevention of orthopaedic implant infection in patients undergoing dental procedures. EvidenceBased Guideline and Evidence Report. Rosemont (IL): AAOS; 2012 [cited 2016 Dec 21]. Available from: http:// www.ada.org/ /media/ADA/Member\%20Center/FIles/ PUDP_guideline.ashx.

22. Wilson WT, Gewitz M, Lockhart PB, Baddour LM, Levison M, Bolger A, CabellCH, TakahashiM, Baltimore RS, NewburgerJW, et al. Prevention of infective endocarditis: guidelines from the American Heart Association: a guideline from the American Heart Association Rheumatic Fever, Endocarditis and Kawasaki Disease Committee, Council on Cardiovascular Disease in the Young, and the Council on Clinical Cardiology, Council on Cardiovascular Surgery and Anesthesia, and the
Quality of Care and Outcomes Research Interdisciplinary Working Group. J Am Dent Assoc 2008 Jan;139 Suppl:3S-24S.

23. Lauber C, Lalh SS, Grace M, Smith MH, MacDougall K, West $\mathrm{P}$, Compton S. Antibiotic prophylaxis practices in dentistry: a survey of dentists and physicians. J Can Dent Assoc 2007 Apr;73(3):245.

24. Jain P, Stevenson T, Sheppard A, Rankin K, Compton SM, Preshing W, Anderson R, Islam S, Mackie AS. Antibiotic prophylaxis for infective endocarditis: knowledge and implementation of American Heart Association Guidelines among dentists and dental hygienists in Alberta, Canada. J Am Dent Assoc 2015 Oct;146(10):743-750.

25. Colterjohn T, de Beer J, Petruccelli D, Zabtia N, Winemaker M. Antibiotic prophylaxis for dental procedures at risk of causing bacteremia among post-total joint arthroplasty patients: a survey of Canadian orthopaedic surgeons and dental surgeons. J Arthroplasty 2014 Jun;29(6):1091-1097.

26. Tong HJ, Hu S, Mok BY, Islam I, Hong CH. Antibiotic prophylaxis prescribing practices of dentists in Singapore. Int Dent J 2014 Apr;64(2):108-114.

27. Zadik Y, Findler M, Livne S, Levin L, Elad S, American Heart Association. Dentists' knowledge and implementation of the 2007 American Heart Association guidelines for prevention of infective endocarditis. Oral Surg Oral Med Oral Pathol Oral Radiol Endod 2008 Dec;106(6):e16-e19.

28. Coutinho AC, Castro GF, Maia LC. Knowledge and practices of dentists in preventing infective endocarditis in children. Spec Care Dentist 2009 Jul-Aug;29(4):175-178.

29. Lockhart PB, Hanson NB, Ristic H, Menezes AR, Baddour L. Acceptance among and impact on dental practitioners and patients of American Heart Association recommendations for antibiotic prophylaxis. J Am Dent Assoc 2013 Sep;144(9): 1030-1035.

30. Tong DC, Rothwell BR. Antibiotic prophylaxis in dentistry: a review and practice recommendations. J Am Dent Assoc 2000 Mar;131(3):366-374.

31. Sollecito TP, Abt E, Lockhart PB, Truelove E, Paumier TM, Tracy SL, Tampi M, Beltrán-Anguilar ED, Frantsve-Hawley J. The use of prophylactic antibiotics prior to dental procedures in patients with prosthetic joints: evidence-based clinical practice guideline for dental practitioners - a report of the American Dental Association Council on Scientific Affairs. J Am Dent Assoc 2015 Jan;146(1):11.e8-16.e8.

32. Deacon JM, Pagliaro AJ, Zelicof SB, Horowitz HW. Current concepts review prophylactic use of antibiotics for procedures after total joint replacement. J Bone Joint Surg Am 1996 Nov;78(11):1755-1770

33. Meehan J, Jamali AA, Nguyen H. Prophylactic antibiotics in hip and knee arthroplasty. J Bone Joint Surg Am 2009 Oct;91(10):2480-2490.

34. Cobb CM, Low SB, Coluzzi DJ. Lasers and the treatment of chronic periodontitis. Dent Clin North Am 2010 Jan;54(1):35-53.

35. Epstein JB, Chong S, Le ND. A survey of antibiotic use in dentistry. J Am Dent Assoc 2000 Nov;131(11):1600-1609.

36. Nelson CL, Van Blaricum CS. Physician and dentist compliance with American Heart Association guidelines for prevention of bacterial endocarditis. J Am Dent Assoc 1989 Feb;118(2):169-173.

37. Marra F, George D, Chong M, Sutherland S, Patrick DM. Antibiotic prescribing by dentists has increased: why? J Am Dent Assoc 2016 May;147(5):320-327. 